\title{
New vaccines enter fray in fight against tuberculosis
}

The global effort to eradicate tuberculosis (TB) may soon gain the tools to succeed. New TB vaccine candidates - the first in more than 80 years-are now in phase 1 clinical trials.

The available bacillus Calmette-Guérin (BCG) vaccine is safe and has been used worldwide for decades. But while it offers children some protection, many adults remain susceptible to pulmonary infection. TB incidence has been exacerbated in recent years by the HIV epidemic and by the rise of drug-resistant strains of Mycobacterium tuberculosis. According to the World Health Organization (WHO), about one-third of the world's population is infected with TB, with 8 million new cases and as many as 3 million deaths each year.

Leading the new effort are two candidates based on the current vaccine. Marcus Horwitz, a microbiologist at the University of California in Los Angeles, has modified BCG to overexpress the $30-\mathrm{kDa}$ protein $\mathrm{A} 85 \mathrm{~b}$, a major secretory protein of M. tuberculosis. In guinea pig models, the recombinant vaccine is more protective than BCG.

Horwitz's research is backed by the US-based Aeras Global Tuberculosis Vaccine Foundation, which will in January begin a phase 1 trial of the vaccine. The foundation has already established a trial site in Cape Town, South Africa, where it is conducting a phase 4 trial of BCG's Tokyo strain to evaluate routes of administration. "We are building the capacity to do large community-based trials," says Larry Geiter, a principal coinvestigator of the trial.

Oxford University researcher Helen McShane is completing safety and immunogenicity trials of a TB subunit vaccine in the Gambia. The MVA85-A vaccine is built from a smallpox virus that is engineered to overexpress the secretory protein $85 \mathrm{~A}$. "The strategy is to boost BCG, rather than replace it," says McShane.

Other groups are using strategies to return the BCG vaccine to its original virulence. In 1999, Marcel Behr, a researcher at McGill University, reported that BCG had lost several genes since its creation in the 1920s (Science $284,1520-1523$; 1999). Behr is now selectively adding back the lost genes in an attempt to prove that the genetic lesions are not just cosmetic, but functional. "We are figuring out where the wounds are and trying to patch them," Behr says. "It is a BCG Band-Aid."

Stewart Cole and his colleagues at the Pasteur Institute-which developed the first $\mathrm{TB}$ vaccine-recently reported successfully restoring several lost BCG genes. When the researchers added back the RD- 1 locus of 11 genes into the vaccine, immunized mice and guinea pigs

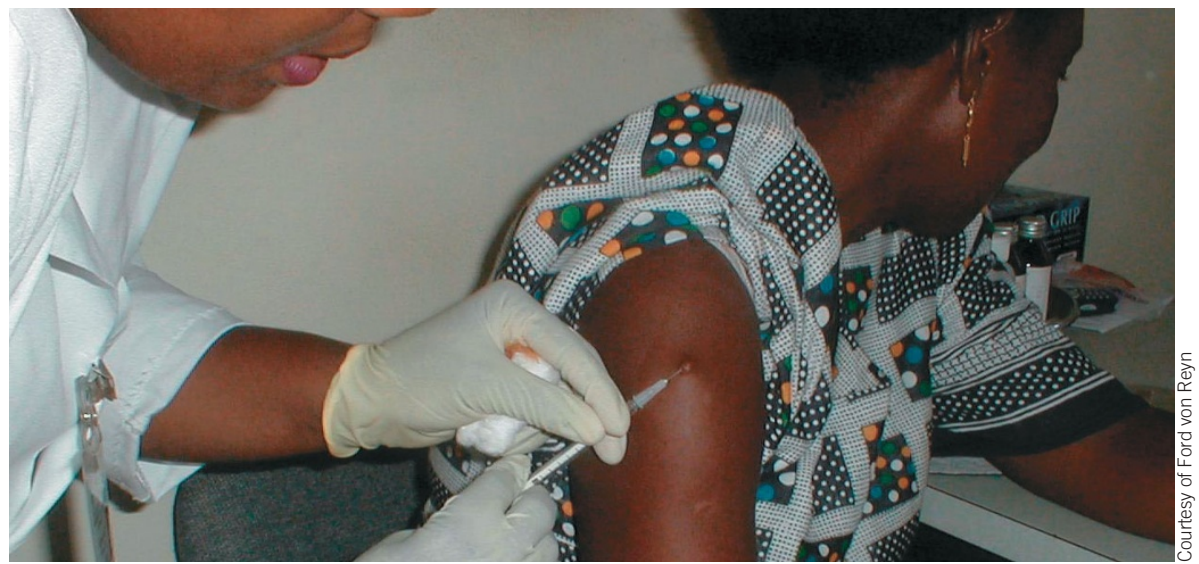

TB or not TB: Clinical trials in Africa are testing new vaccines against tuberculosis

showed better protection against infection.

A critical element in the fight against TB is coinfection with HIV. Ford von Reyn, professor of medicine at Dartmouth, and his colleagues have developed a TB booster that is safe in HIVpositive patients. The multidose, killed vaccine doesn't adversely affect viral load or CD4 count, and increases cellular immune response and production of interferon- $\gamma$, says von Reyn.

The group is in its third year of a National Institutes of Health (NIH)-sponsored efficacy trial in Tanzania, where researchers have enrolled 2,300 BCG-vaccinated, HIV-positive people with CD4 counts above 200. "We are trying to identify a strategy that would be applicable in the near term, not ten years from now," says von Reyn.

Funding for TB research had been limited until the WHO in 1993 declared it a global public health emergency. Between 1991 and 2001, the NIH budget for TB researchers increased from \$3.6 million per year to \$56 million. Aeras, which has recently shifted its focus from research to development, received \$25 million from the Bill and Melinda Gates Foundation.

Hannah Hoag, Montreal

\section{Antibiotic resistance soars in Europe}

Responding to "worrying trends" in antimicrobial resistance in Europe, the European Union has announced two new projects, totaling \$16 million, to study how resistance arises.

There is increasing incidence of Escherichia coli strains resistant to third-generation cephalosporins, a consistent rise in fluoroquinolone resistance, and resistance rates of more than $50 \%$ to ampicillin, according to a new report from the European Antimicrobial Resistance Surveillance System (EARSS), an infectious disease network of 28 countries.

Resistance to third-generation cephalosporins has remained lower than $6 \%$ in most countries. But higher rates in some eastern European countries are associated with the spread of strains producing $\beta$-lactamases, enzymes that can destroy aminopenicillins such as ampicillin. The EARSS also reports a "consistent and marked" rise in fluoroquinolone-resistant $E$. coli in many European countries. Rates of resistance are currently about $10 \%$, but eight countries experienced a 1.5 -fold increase in just two years.

Resistance in E. coli to traditional antibiotics such as aminopenicillins is now common in Europe, with only Finland and Sweden reporting rates of less than $30 \%$. Among the 25 countries surveyed, resistance rates varied from $25 \%$ to $64 \%$. More than half of all strains isolated from bloodstream infections harbor plasmids that encode $\beta$ lactamases. The report is based on routinely generated antimicrobial susceptibility data from 700 laboratories and an estimated sample population of 100 million.

EARSS monitors resistance in several other major pathogens, including Staphylococcus aureus, Streptococcus pneumoniae, Enterococcus faecalis and E. faecium, but it singles out $E$. coli resistance as showing the most worrying trends. Multiresistant bacteria pose a "potentially serious impediment" to transplant surgery, cancer therapy and treatment of infections, warns Hajo Grundmann, project leader for the EARSS. Tony Sheldon, Utrecht 